\title{
Mapping the carbon monoxide coma of comet 29P/Schwassmann-Wachmann 1
}

\author{
M. Gunnarsson ${ }^{1,2}$, D. Bockelée-Morvan ${ }^{1}$, N. Biver ${ }^{1}$, J. Crovisier $^{1}$, and H. Rickman ${ }^{2}$ \\ ${ }^{1}$ Laboratoire d'Études Spatiales et d'Instrumentation en Astrophysique (LESIA), Observatoire de Paris, CNRS, UPMC, \\ Université Paris Diderot, 5 place Jules Janssen, 92190 Meudon, France \\ e-mail: marcusgun@gmail.com \\ 2 Astronomiska Observatoriet, Box 515, 75120 Uppsala, Sweden
}

Received 12 June 2007 / Accepted 27 December 2007

\begin{abstract}
Context. CO is assumed to be the main driver behind the activity of comet 29P/Schwassmann-Wachmann 1, which resides in a nearcircular orbit at $6 \mathrm{AU}$ from the Sun. Several properties of the outgassing of CO can be deduced from its millimetre-wave emission. Earlier studies have indicated $\mathrm{CO}$ production from the nucleus as well as an extended source.

Aims. We have sought to further investigate the nature of the CO production in comet 29P/Schwassmann-Wachmann 1 , through the use of newly available instrumentation.

Methods. We used the HERA receiver array on the 30-m IRAM telescope to map the $230 \mathrm{GHz} \mathrm{CO}(J=2-1)$ line in the comet with an unprecedented sensitivity and spatial coverage, and a high spectral resolution ( $20 \mathrm{kHz}$, i.e., $25 \mathrm{~m} \mathrm{~s}^{-1}$ ). A 36-point map, 60 by $60^{\prime \prime}$, was obtained in June 2003, and a 25-point map, 96 by 96", in January 2004.

Results. The $\mathrm{CO}$ emission line has a characteristic asymmetric profile. Our analysis is based on a coma model, where the outgassing pattern is derived from the shape of this line at the central position of the map. When comparing to the observations, both maps show a line intensity at offset positions that is $2-3$ times stronger than the model prediction. Different explanations for this are evaluated, and it is found that for the global coma character, an extremely low gas temperature in the inner coma reproduces the observed radial profile. A cold inner coma depletes the population of the $\mathrm{CO} J=2$ rotational level in the region closest to the nucleus, making spectra observed at offset positions relatively stronger. From the global appearance of the maps, the coma was found to be largely axisymmetric, and the presence of a strong extended source of $\mathrm{CO}$, as indicated from earlier observations using the SEST telescope, was not seen. When examining the maps in more detail, a possible exception to this was seen in an area $\sim 30^{\prime \prime}$ south of the comet, where an excess in emission is present in both maps. Model fits to the spectra based on the cold inner coma that we find, with an intital kinetic temperature $T_{\text {kin }}=4 \mathrm{~K}$, give a measure of $Q_{\mathrm{CO}}$, the CO production rate. $Q_{\mathrm{CO}}$ was found to be $(3.9 \pm 0.2) \times 10^{28} \mathrm{~mol} \mathrm{~s}^{-1}$ in June 2003, and $(3.7 \pm 0.2) \times 10^{28} \mathrm{~mol} \mathrm{~s}^{-1}$ in January 2004. These values are a factor $\sim 1.5$ higher than that derived using only the information available from non-mapped data, and this adjustment applies also to previously published production rates.
\end{abstract}

Key words. comets: individual: 29P/Schwassmann-Wachmann 1 - radio lines: solar system - methods: data analysis

\section{Introduction}

Comet 29P/Schwassmann-Wachmann 1 (hereafter SW1) has been observed through its millimetre-wave emission from carbon monoxide for over a decade since the first detection in 1993 (Senay \& Jewitt 1994). In these radio spectra, the emission line has exhibited a distinct, asymmetric shape, indicative of CO being released from the nucleus primarily toward the Sun, at a velocity of around $0.5 \mathrm{~km} \mathrm{~s}^{-1}$ (Crovisier et al. 1995; Biver 1997; Festou et al. 2000). The emission line also covers positive velocities, showing the existence of molecules moving away from the Sun at around $0.3 \mathrm{~km} \mathrm{~s}^{-1}$.

The release of volatile gases in distant comets is believed to happen as amorphous water ice undergoes crystallization. Through simulations, this process is generally found to occur at some depth inside the nucleus (Tancredi et al. 1994; Cabot et al. 1996), with the released gases diffusing outward through the porous material. For porous ice, the skin depth connected to diurnal heat variations is shallow, and therefore gas release deep inside the nucleus should result in isotropic outgassing, as the day/night heat variations level out before reaching the crystallization region. One idea to explain the strongly anisotropic outgassing that is observed in comet SW1 is a pole-on viewing geometry, allowing for a deep heat contrast between the day- and nightside. However, in all observations since the first detection, that is equivalent to more than 70 percent of an orbital period, the appearance of the line has remained similar. Thus, its shape cannot be explained by a pole-on orientation. Rather, the mechanism releasing $\mathrm{CO}$ from the nucleus must occur on or close to the nuclear surface in order to be concentrated near the subsolar point. This requirement arises also, as found in modelling by Enzian et al. (1997), when considering CO as a driver of the outburst phenomena, for which SW1 is famous. Efficient surface erosion is likely a necessity for such a condition to persist.

In observations using the Swedish-ESO Submillimetre Telescope (SEST) at ESO, Chile, obtained in 1998 (Gunnarsson et al. 2002), a simple map of the $\operatorname{CO}(J=2-1)$ emission was created for offset distances of up to $22^{\prime \prime}$. In an outgassing scenario where all $\mathrm{CO}$ is produced from the nucleus, the intensity of the emission line should decline rapidly with offset distance. Instead, the line area remained roughly constant over the whole 
map, suggesting that part of the $\mathrm{CO}$ in the coma was being produced from an extended source.

As the characteristics of the extended source were difficult to determine from these observations, more observations were required to produce a map covering a larger angular area. The multi-receiver array HERA ${ }^{1}$ (Schuster et al. 2004) is installed on the IRAM ${ }^{2} 30-\mathrm{m}$ telescope on Pico Veleta, Spain. With this instrument, the 30-m telescope is well suited to observing the $\mathrm{CO}(J=2-1)$ line at $230 \mathrm{GHz}$ in comets. With HERA, nine map positions on a square grid, separated by $24^{\prime \prime}$, are observed simultaneously. The advent of this instrument is timely, as the position of the comet on the sky has progressed toward favourable elevations for observations from the northern hemisphere. We observed comet SW1 using HERA on two occasions, in June 2003, and January 2004.

\section{Observations}

Between June 26 and 29, 2003, the HERA instrument was used to create a 36-point map of the coma of comet SW1 through the $\mathrm{CO}(J=2-1)$ emission line, at rest frequency $230.538000 \mathrm{GHz}$. The map points were spaced $12^{\prime \prime}$ apart on a regular grid, a spacing equal to the half-power beamwidth (HPBW) of the HERA instrument at this frequency. The main beam efficiency was 0.52 , and the observing mode was frequency switching with a throw of $6.9 \mathrm{MHz}$. The telescope is equipped with a backend autocorrelator spectrometer called VESPA ${ }^{3}$, with a total of 18000 channels, i.e. 2000 channels per receiver in the HERA instrument. The data were collected at the maximum resolution of VESPA, a channel spacing of $20 \mathrm{kHz}$, which corresponds to $25 \mathrm{~m} \mathrm{~s}^{-1}$ at $230 \mathrm{GHz}$. The receiver array was aligned with one axis along the Sun-comet line on the sky. In a single-beam situation where each map point is observed in sequence over the course of several days, temporal changes must be considered when analyzing the data. The simultaneous observation of nine map positions was a very important factor for this project, as temporal changes in the coma structure can thus be ruled out as an explanation for any phenomena seen in the map. The observed map is shown in Fig. 1. The light grey background is the sky plane, and each small spectrum is shown centred on the corresponding map position. In the spectra, the main beam brightness temperature $\left(T_{\mathrm{B}}\right)$ is shown versus velocity $\left(\mathrm{km} \mathrm{s}^{-1}\right)$. In the figure, the Sun is to the left. The elongation was $112^{\circ}$, the phase angle was $9^{\circ}$ and the receiver array was tilted by $24^{\circ}$ with respect to the RA-Dec coordinate system.

The emission line has its maximum intensity at the centre position in the map. This is the expected case for an expanding coma, and the new map thus differs from the SEST observations obtained in 1998 (Gunnarsson et al. 2002). Further inspection shows that emission from the comet is present also in points along the edges of the map. Since our goal was to investigate the nature of all $\mathrm{CO}$ production in the comet, we wished to map the coma to the point where the $\mathrm{CO}$ emission would not be visible.

To improve on the data, and to cover a larger angular area, the comet was observed again between January 14 and 16, 2004. 25 map positions were observed, with a spacing of $24^{\prime \prime}$, as shown in Fig. 2. This time the Sun was to the right in the frame of the map, which was tilted by $27^{\circ}$ with respect to the RA-Dec system. The elongation was $47^{\circ}$ and the phase angle was $7^{\circ}$. The parameters characterizing the instrument were unchanged.

\footnotetext{
1 HEterodyne Receiver Array.

2 Institut de Radioastronomie Millimétrique.

3 VErsatile SPectrometer Assembly.
}

A $\log$ of the observations is given in Table 1. For the June 2003 data, high wind speeds resulted in the loss of some of the allocated time. The different amount of integration time listed for the centre position in the two observing runs is an effect of the map layout. In the 2003 observation, the 36-point map was created by cycling the position of the HERA instrument through four points offset by $12^{\prime \prime}$. This resulted in an equal integration time for all map points, which is $1 / 4$ of the total time. In the 2004 observation, the instrument position was also cycled through four positions, but always with a corner point of the instrument at the centre of the map. This way, the integration time for the centre position was equal to the total time. For another 8 points where the footprint of the instrument overlapped, the integration was $1 / 2$ of the total, and for the remaining points, $1 / 4$ of the total time.

One special instance of post-processing was necessary for these two datasets. During the time when the two observing runs were performed, an error was present in the software controlling the $1 \mathrm{~mm}$ receiver in narrow bandwidth mode (which we used). This mode incorporates a $256 \mathrm{MHz}$ frequency offset, and because of the error the sky frequency was not corrected for the Doppler shift to this offset from the heliocentric velocity of the Earth (G. Paubert, pers. comm. 2006). The net effect is that the velocity scale in the observed spectra was inaccurate by $+31 \mathrm{~m} \mathrm{~s}^{-1}$ for 2003 and $-24 \mathrm{~m} \mathrm{~s}^{-1}$ for 2004 . This error has been corrected in all data presented here, by shifting the spectra one channel (of channel width $25 \mathrm{~m} \mathrm{~s}^{-1}$ ) toward negative velocities for 2003 and one channel toward positive velocities for 2004 . The remaining errors of +6 and $+1 \mathrm{~m} \mathrm{~s}^{-1}$ are deemed insignificant in this analysis.

In all figures showing spectra in the present paper, the data were smoothed (downsampled) once for clarity. The resulting channel width is $50 \mathrm{~m} \mathrm{~s}^{-1}$.

The area of the emission line at the centre position shows that the activity level of the comet was the same on the two occasions. The line areas were $0.181 \pm 0.009 \mathrm{~K} \mathrm{~km} \mathrm{~s}^{-1}$ (Jun. 2003), and $0.143 \pm 0.004 \mathrm{~K} \mathrm{~km} \mathrm{~s}^{-1}$ (Jan. 2004) in brightness temperature scale. Although these areas were different, so was $\Delta$ on the two occasions. As a first approximation the line area $A_{\ell}$ is proportional to the $\mathrm{CO}$ column density, which is proportional to $Q_{\mathrm{CO}} / \Delta$ since the aperture subtends a distance at the comet that is much smaller than the $\mathrm{CO}$ scalelength. Thus, $Q_{\mathrm{CO}} \propto A_{\ell} \times \Delta$, and the production rate was nearly identical in June 2003 and Jan. 2004. It is also of interest to compare the line areas to that of the 1998 observations done at SEST. Compensating for the different distances and telescope parameters, we note that the number of $\mathrm{CO}$ molecules inside the telescope beam was comparable in 1998, 2003 and 2004.

From inspection of Figs. 1 and 2, and comparing spectra at corresponding points in the maps, the observations appear very similar on the two occasions. Figure 3 shows a closer comparison of the line at the centre position for the two dates. In order to see a well defined shape in both lines, the noise has been reduced in the 2003 dataset by co-adding data from the five centremost points (nucleus-centred and the four points at $12^{\prime \prime}$ offset). There is no noticeable difference in the two line profiles. The velocity shift of the blueshifted peak, and also of the smaller redshifted peak, did not change, and the overall shape of the profile is also the same.

Simultaneously to our IRAM observations of June 2003, a search for the $1_{10}-1_{01}$ line of water at $557 \mathrm{GHz}$ was undertaken with the Odin satellite (Biver et al. 2007). This was an effort to look for water outgassing from heated icy grains. A marginal detection, possibly obtained during the first part of the 


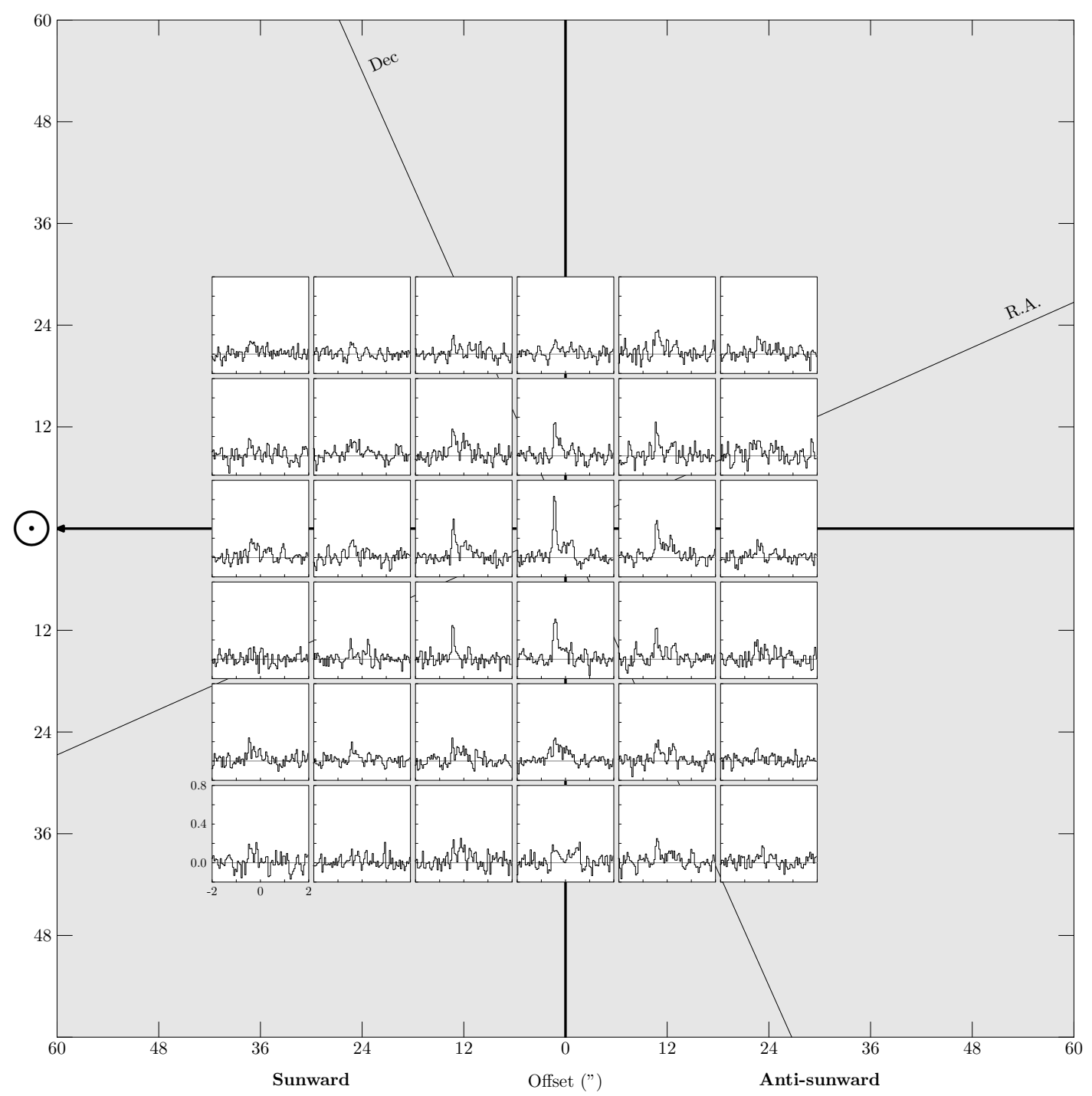

Fig. 1. The $\operatorname{CO}(J=2-1)$ map of comet SW1 from June 2003. The map points are separated by 12 ", equal to one HPBW beam diameter of the HERA instrument. Each box is centred on the map position where the spectrum was recorded. The axes in each small spectrum are main beam temperature $(\mathrm{K})$ versus velocity $\left(\mathrm{km} \mathrm{s}^{-1}\right)$, with scales as shown for the bottom left corner box. The maps are aligned along the Sun-comet line on the sky. The direction toward the Sun is marked by a $\odot$ sign. The RA-Dec coordinate system is also indicated (thinner tilted lines). $10^{\prime \prime}$ on the sky plane is equivalent to $3.8 \times 10^{4} \mathrm{~km}$ at the location of the comet.

Table 1. $\log$ of the $\operatorname{CO}(J=2-1)$ observations of comet SW1 performed using HERA at the $30 \mathrm{~m}$ telescope.

\begin{tabular}{llllrr}
\hline \hline \multirow{2}{l}{ Date (UT) } & $\begin{array}{l}r_{\mathrm{h}} \\
(\mathrm{AU})\end{array}$ & $\begin{array}{l}\Delta \\
(\mathrm{AU})\end{array}$ & $\begin{array}{r}\text { Integ }^{1} \\
(\mathrm{~min})\end{array}$ & $\begin{array}{r}\mathrm{rms}^{2} \\
(\mathrm{mK})\end{array}$ \\
\hline 2003 & June 26 & 5.75 & 5.31 & 15 & 92 \\
& June 27 & 5.75 & 5.30 & 65 & 53 \\
& June 28 & 5.75 & 5.28 & 15 & 107 \\
& June 29 & 5.75 & 5.27 & 15 & 74 \\
2004 & Jan. 14 & 5.73 & 6.34 & 84 & 33 \\
& Jan. 15 & 5.73 & 6.35 & 136 & 33 \\
& Jan. 16 & 5.73 & 6.37 & 180 & 17 \\
\hline
\end{tabular}

1. Total time spent on the centre position. See text.

2. Rms of noise in $T_{\mathrm{A}}$ (antenna temperature) for the centre position at a resolution of $20 \mathrm{kHz}$.

observation, could not be confirmed later. The upper limit of the water production was estimated as $Q\left[\mathrm{H}_{2} \mathrm{O}\right]<2.5 \times 10^{28} \mathrm{~s}^{-1}$.

\section{Interpretation and results}

\subsection{The nucleus-centred spectrum}

In both datasets, the centre spectrum has a characteristic asymmetric shape which appears to be a permanent feature in comet SW1. Its strong narrow peak at negative velocities is clearly the signature of $\mathrm{CO}$ molecules originating from or near the subsolar point on the nucleus, and their velocity can be accurately determined from the velocity shift of this peak. However, sophisticated modelling (Crifo et al. 1999) predicts a higher velocity for this flow of $\mathrm{CO}$ - over $0.6 \mathrm{~km} \mathrm{~s}^{-1}$ in contrast to the $0.45-0.50 \mathrm{~km} \mathrm{~s}^{-1}$ measured in the many mm-wave observations performed over the last decade. A possible explanation for this could be mass loading - i.e. the presence of another, heavier, molecular species that is released together with the $\mathrm{CO}$, and which reduces the velocity of the flow. A candidate molecule for this could be $\mathrm{CO}_{2}$, which is not visible at radio wavelengths, but may be present in SW1. To match the observed dayside terminal velocity, the $\mathrm{CO}_{2}$ production must exceed that of $\mathrm{CO}$ by a factor of $\sim 4$. Using the present data, the presence of such an amount 


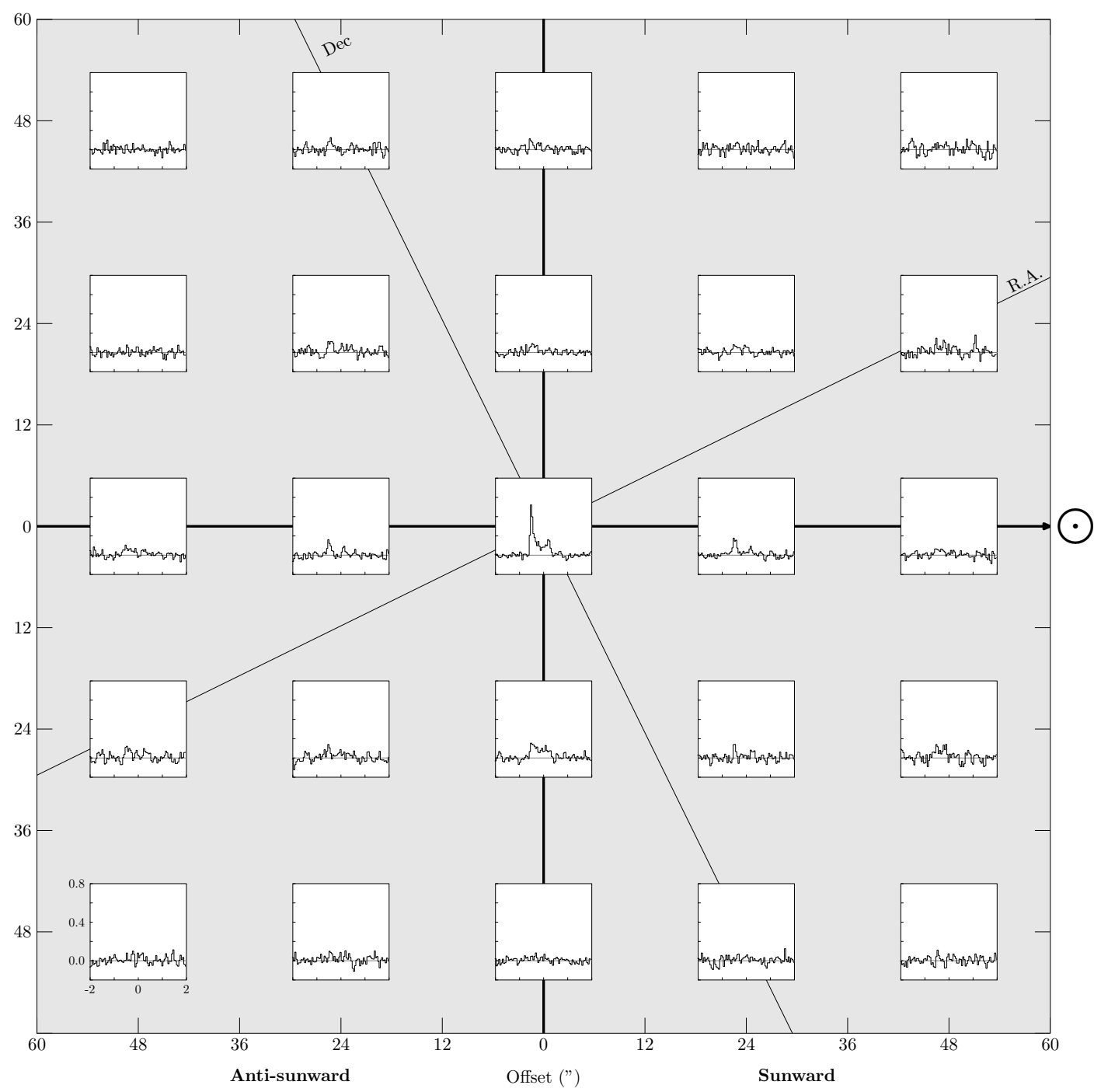

Fig. 2. The $\mathrm{CO}(J=2-1)$ map of comet SW1 from January 2004. Here the map points are separated by $24^{\prime \prime}$, equal to two HPBW beam diameters. The axes, alignment and angular map scale are the same as for Fig. 1. The direction toward the Sun was opposite to that of the 2003 map. $10^{\prime \prime}$ on the sky plane is equivalent to $4.6 \times 10^{4} \mathrm{~km}$ at the location of the comet.

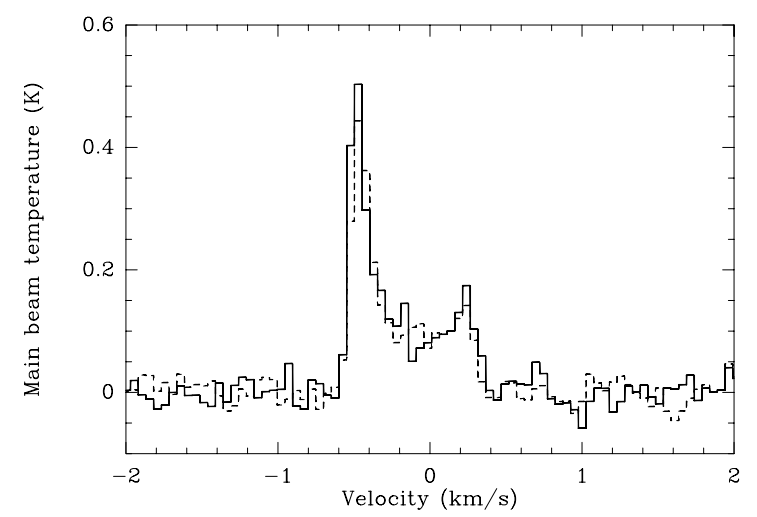

Fig. 3. Comparison of the line shape between the two observing runs. For the 2003 data (dashed line), an average of the five centremost spectra is shown, in order to reduce the noise. This is compared to the centremost spectrum of the 2004 data (solid line). The appearance of the two lines, as defined by the velocity of the blue- and redshifted peaks, is nearly identical.

of $\mathrm{CO}_{2}$ in the coma of SW1 cannot be ruled out, as detailed in Sect. 3.2.3.

\subsection{Global character of the coma}

In order to analyze the spectra in the maps, we first examine the global behaviour of the $\mathrm{CO}$ line, by studying how the emission line changes with projected radius from the nucleus-centred position. Therefore we compute radial averages, by co-adding all spectra inside annuli of increasing radius. The resulting averages are used to study the global appearance of the $\mathrm{CO}$ coma. The integrated line areas are given for both datasets in Table 2, and the corresponding lines are shown in Fig. 4. While we wanted to map a region large enough to see all $\mathrm{CO}$ emission in the coma (i.e. no visible emission at the edge of the map), Fig. 4 shows that a detection is present also at the largest projected distance (the corner points) of the 2004 map.

\subsubsection{Coma model}

When interpreting the spectra, a Monte Carlo coma model is used, as described in Festou et al. (2000) and more detailed in Gunnarsson et al. (2002).

The coma model produces a simulated emission line for given values of $r_{\mathrm{h}}, \Delta$, phase angle and telescope parameters. The 

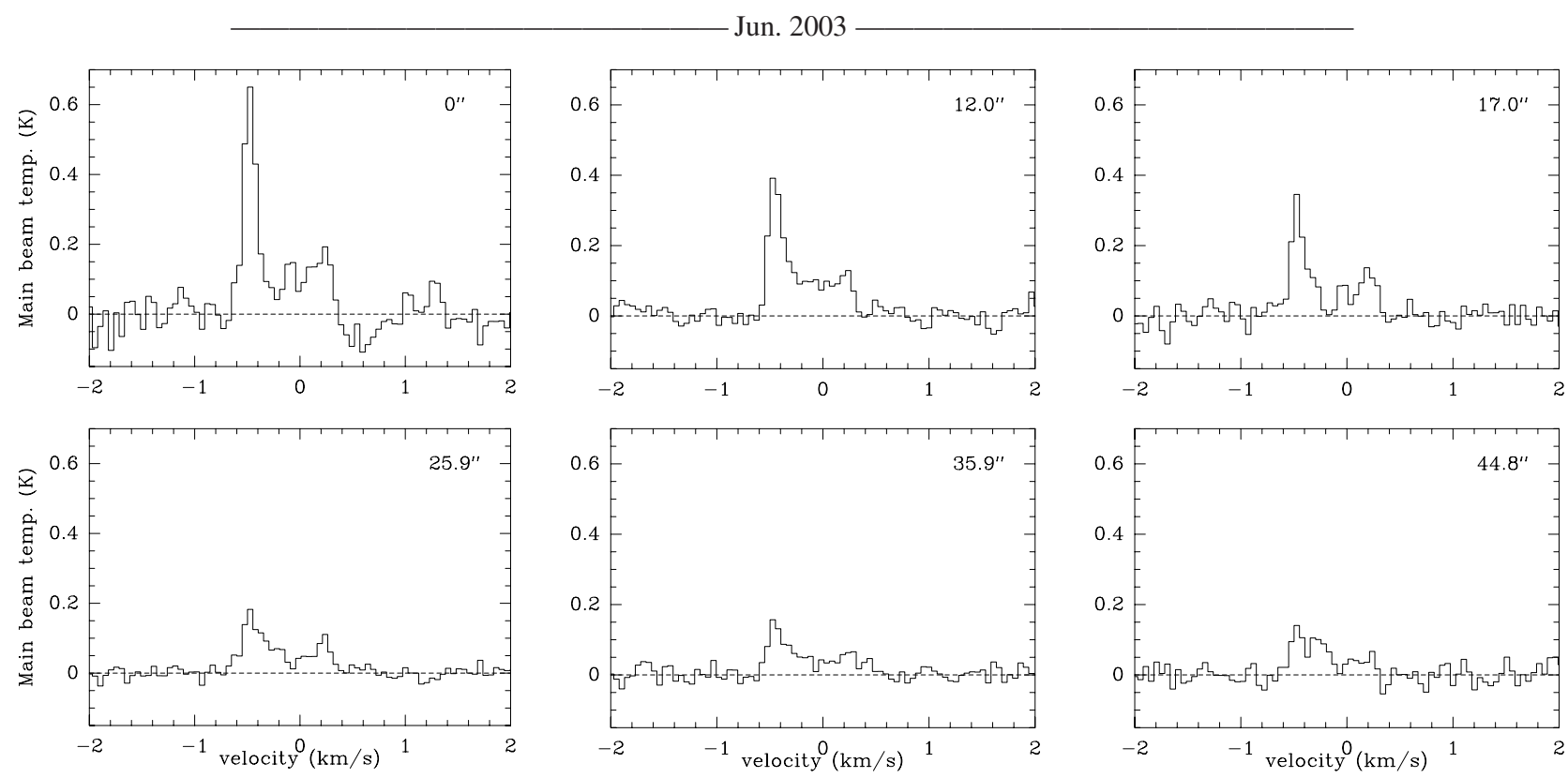

Jan. 2004
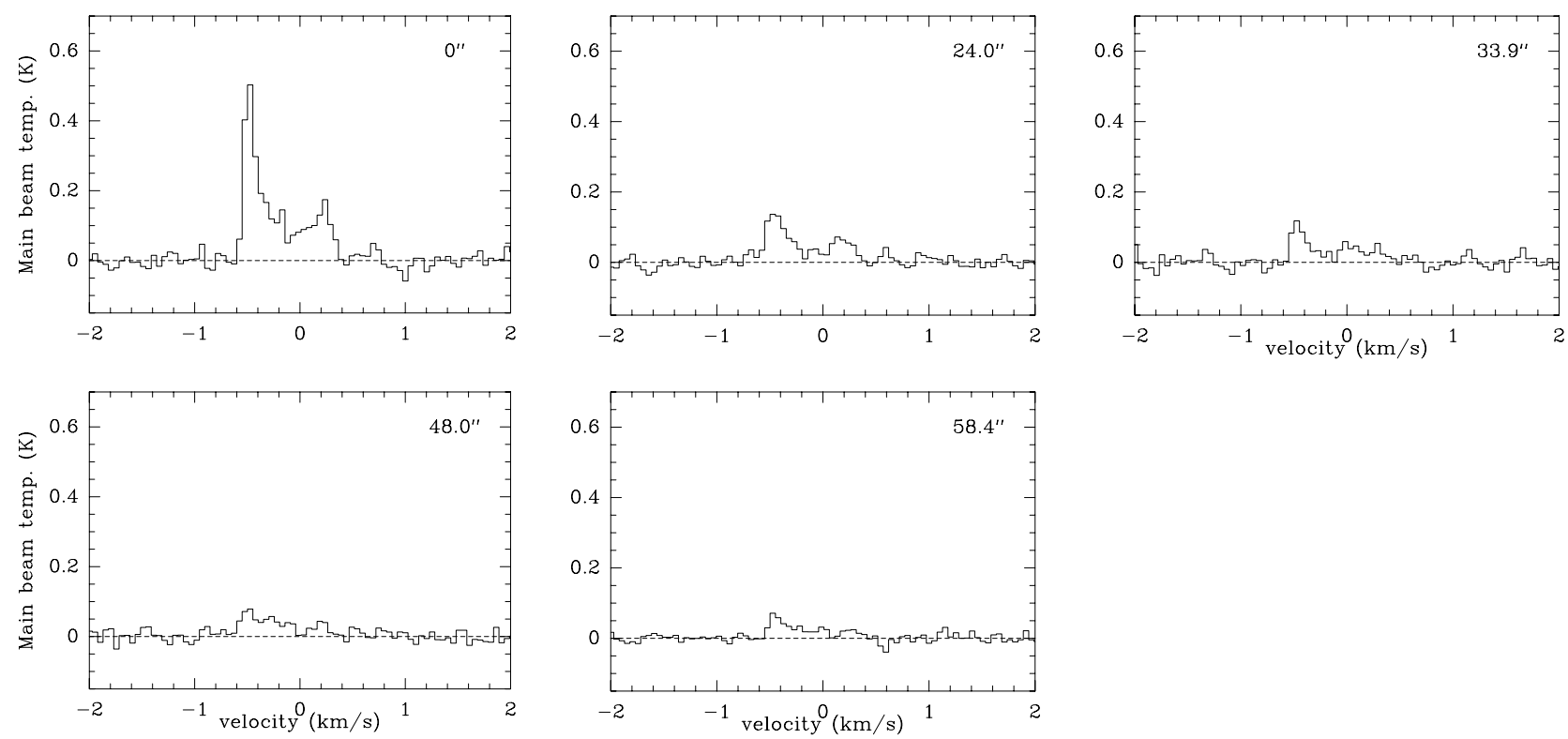

Fig. 4. Radially averaged spectra from the two datasets. Each spectrum is the sum of all spectra inside a radial interval, of which the mean radius is shown in the top right corner of each box.

Table 2. Average line areas at increasing offset radii, for the 2003 and 2004 datasets. Main beam brightness temperatures are given. The mean velocity shift of the lines are also listed.

\begin{tabular}{llll}
\hline \hline & Radius (") & $\begin{array}{l}\text { Line area } \\
\left(\mathrm{K} \mathrm{km} \mathrm{s}^{-1}\right)\end{array}$ & $\begin{array}{l}\text { Mean velocity } \\
\left(\mathrm{km} \mathrm{s}^{-1}\right)\end{array}$ \\
\hline 2003 & 0 & $0.181 \pm 0.009$ & $-0.253 \pm 0.018$ \\
& 12.0 & $0.116 \pm 0.005$ & $-0.249 \pm 0.016$ \\
& 17.0 & $0.097 \pm 0.006$ & $-0.243 \pm 0.022$ \\
& 25.9 & $0.071 \pm 0.003$ & $-0.226 \pm 0.015$ \\
& 35.9 & $0.051 \pm 0.004$ & $-0.180 \pm 0.024$ \\
& 44.8 & $0.059 \pm 0.006$ & $-0.249 \pm 0.033$ \\
\hline 2004 & 0 & $0.143 \pm 0.004$ & $-0.232 \pm 0.010$ \\
& 24.0 & $0.053 \pm 0.003$ & $-0.222 \pm 0.019$ \\
& 33.9 & $0.041 \pm 0.003$ & $-0.200 \pm 0.026$ \\
& 48.0 & $0.030 \pm 0.003$ & $-0.280 \pm 0.038$ \\
& 58.4 & $0.021 \pm 0.002$ & $-0.196 \pm 0.038$ \\
\hline
\end{tabular}

shape of the model line depends on the outgassing pattern of the simulated comet, which is set up using a limited set of model parameters. A basic assumption is that the outgassing locally depends on $\mu$, an entity which is the cosine of the solar zenith angle on the nuclear dayside, and zero on the nightside. In this way, two model parameters are used to describe a continuous asymmetric velocity field, by setting the subsolar and night side outgassing velocity. Another two parameters set the CO production rate, and the contrast in production between the day and night sides. The kinetic temperature $T_{\mathrm{k}}$ determines the broadening of the line, as well as the initial excitation of the molecules. When the molecules travel outward, their rotational distribution gradually changes, as molecular collisions become less frequent. Far from the nucleus, the molecules are said to be in fluorescence equilibrium. The excitation is then governed only by pumping from the Solar IR radiation. This model has also been refined to 


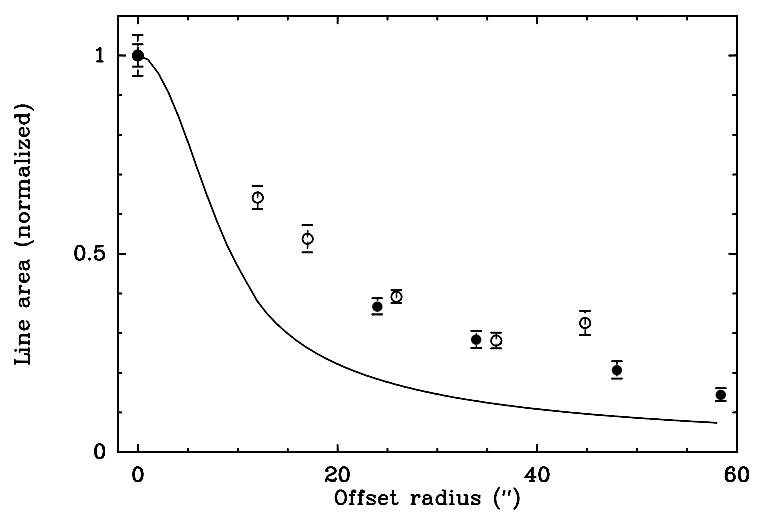

Fig. 5. Normalized line area of the radial average spectra for 2003 (open circles), and 2004 (filled circles) compared to that predicted by a model (solid line) assuming nuclear CO production, with an initial coma temperature of $8 \mathrm{~K}$.

include other elements, such as $\mathrm{CO}$ production from a distributed source.

We performed a model fit assuming nuclear CO production, based only on the shape of the centremost line profile. We set the subsolar and nightside velocities to 0.5 and $0.3 \mathrm{~km} \mathrm{~s}^{-1}$, respectively, and the $\mathrm{CO}$ production contrast was set to $1 / 5$ (this number is the ratio of the total productions on the night- and day-side). Assuming pure thermal broadening, the width of the blueshifted peak corresponds to $8.5 \pm 0.6 \mathrm{~K}$, and we selected $8 \mathrm{~K}$ for the kinetic temperature of the gas in the inner coma. The area of the line generated by the model is shown as a function of projected radial distance in Fig. 5. The observed line areas from the two observing runs are shown in the same figure for comparison. Both the model line and the observed line areas are shown normalized to the centre position. As $\Delta$ was different on the two occasions, the offset angle translates into different physical distances at the comet $-24^{\prime \prime}$ is equivalent to $0.92 \times 10^{5} \mathrm{~km}$ for 2003 and $1.10 \times 10^{5} \mathrm{~km}$ for 2004 . However, as this absolute distance is proportional to $\Delta$, so also is the physical diameter of the $11^{\prime \prime}$ telescope beam. Because of this, the falloff in model line area versus offset angle given in arcsec is almost identical for the two occasions. This allows comparison of all the observed data points to the model prediction in the same diagram.

It is evident from Fig. 5 that this first model approach, with its simple assumptions about the outgassing pattern, cannot explain the data, as the line area at large offset radii is about a factor of 2-3 larger than that predicted by the model.

\subsubsection{Extended CO source}

A first step in finding an explanation for the observed line intensities is to consider the idea used to explain the 1998 observations, i.e., an extended CO source consisting of solid icy grains of water ice that also contain CO. This scenario could explain the line shapes seen in the SEST observations, and such an extended source is necessary in a case where the observed intensity remains unchanged over a region containing many map points. In the current observations, a cloud of $\mathrm{CO}$-producing material surrounding the nucleus would result in a less steep decrease in line intensity, as observed. However, the presence of such a source influences the shape of the emission line. The extended source material would consist of icy grains, and to retain their icy component for some time, they cannot be too small (sizes should be on the order of $10 \mu \mathrm{m}$ ), and then their velocity cannot be more

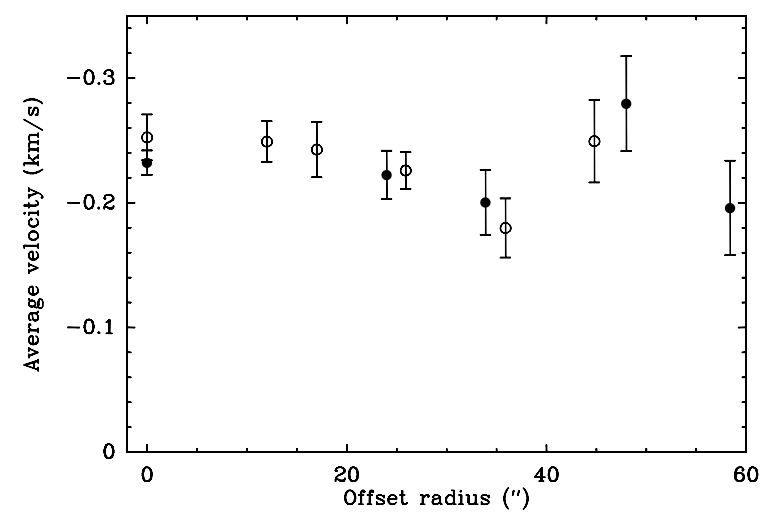

Fig. 6. Average velocity shift of the CO $230 \mathrm{GHz}$ line observed in 2003 (open circles) and 2004 (filled circles), as a function of radial offset.

than a fraction of the gas velocity (Crifo \& Rodionov 1997). The grains would thus travel slowly in the coma, and produce $\mathrm{CO}$ through isotropic outgassing from each grain. The contribution to the emission line from this source would then have a symmetric shape.

When observing the spectra resulting from such a scenario, moving from the centre position to offset positions, the blueshifted peak, which results from nuclear CO outgassing, drops in intensity quicker than the remainder of the line where CO from the extended source contributes. Then, the overall shape of the emission line would change to an increasingly symmetric shape with increasing offset radius. The asymmetry of the line is quantified through calculation of the average velocity shift of the observed lines. If isotropic outgassing from grains was dominant at large radii, we would see a decrease in the mean velocity shift of the line. As shown in Fig. 6, this is not the case for either the 2003 or 2004 observations. Rather, the velocity shift remains roughly unchanged. This means that the line shape at offset positions is a scaled-down copy of that at the centre, a result that cannot be reproduced through the icy grain scenario, at least for the global case.

When applying the above analysis to the spectra observed at SEST in 1998, a marked decrease in velocity shift is seen for increasing radius, showing that the 1998 observations, where $\mathrm{CO}$ production of an extended character dominated the map, presented conditions much different from what we see in the present data sets.

\subsubsection{Parent molecule}

In this look at the global characteristics, the centre position constitutes a pronounced maximum in intensity, and the offset spectra are scaled-down versions of the centre spectrum, although stronger than expected. A possible explanation for this is another type of extended $\mathrm{CO}$ source, where the $\mathrm{CO}$ produced in the coma follows the flow of gas released from the nucleus. The mechanism in this case would be the photolysis of a parent, forming $\mathrm{CO}$ as a daughter molecule. When including the hypothetical parent molecule in the coma model, two parameters are used to define its behaviour; the production rate $Q_{\mathrm{p}}$ of the parent, and its lifetime in seconds. Considering the line shape, which has a distinct peak also at large offset radii, the velocity of the fragment CO molecules relative to the parent should be small, otherwise the peaked shape would be distorted. This parameter is set to zero in the model. Using this model, a fit to the data, both in terms of shape and intensity of the line, can be attained using 
a CO-to-parent mixing ratio of 1:2.5 in the nuclear outgassing, and a lifetime of $10^{4} \mathrm{~s}$ at $1 \mathrm{AU}$ (or $3 \times 10^{5} \mathrm{~s}$ at $5.7 \mathrm{AU}$ ).

The problem with a hypothetical parent molecule as an explanation is that no good candidate is known. $\mathrm{H}_{2} \mathrm{CO}$ does dissociate to form $\mathrm{CO}$, but this species, among others, has not been detected, and the upper limit found for its abundance in SW1, of less than one percent of CO, rules it out (Biver 1997).

One obvious candidate to consider is $\mathrm{CO}_{2}$. This molecule has no electric dipole moment, and is thus not observable through radio lines. It is thus undetected so far in comet SW1, but may well be present in the outgassing. Indeed, at the heliocentric distance of $6 \mathrm{AU}$, the temperature of the sunlit hemisphere of SW1 is expected far above the sublimation temperature of $\mathrm{CO}_{2}$ ices $(72 \mathrm{~K})$. In comet Hale-Bopp, the $\mathrm{CO}_{2} / \mathrm{CO}$ abundance ratio was $~ 30 \%$ at 3 AU from the Sun (Crovisier et al. 1997). CO-ice being more volatile than $\mathrm{CO}_{2}$-ice, a higher relative $\mathrm{CO}_{2}$ production in $\mathrm{SW} 1$ that would result from selective depletion of the most volatile species would not be unexpected. However, at 6 AU from the Sun, $\mathrm{CO}_{2}$ has a lifetime that is two orders of magnitude greater than that of the hypothetical molecule discussed above, which would require a high production rate $\left(\mathrm{a}\right.$ few $10^{30} \mathrm{~mol} \mathrm{~s}^{-1}$ ) to fit the data. While the long lifetime of $\mathrm{CO}_{2}$ is the one reason speaking against this molecule being the parent, another issue is in the ejection velocity of the daughter molecules. It is required that this velocity is very low, in order not to distort the peaked line shape. Already at small ejection velocities, the observed line profile has a broadened shape with wide "wings", rather than the sharp peak we observe. The spectral signature of a daughter molecule is explained e.g. in Bockelée-Morvan et al. (1990).

In their study of solar photo rates, Huebner et al. (1992) found an excess energy of $4.3 \mathrm{eV}$ for the main photolysis channel $\mathrm{CO}_{2} \rightarrow \mathrm{CO}+\mathrm{O}\left({ }^{1} D\right)$. If this energy was fully transmitted as translational energy to the fragments, this would lead to a mean ejection velocity of $3.3 \mathrm{~km} \mathrm{~s}^{-1}$ for CO. However, only part of this energy goes to translational energy, the remaining going to rotational and vibrational energy. So, this velocity must be considered as an upper limit.

Miller et al. (1992) published laboratory data on the production of $\mathrm{CO}$ following photodissociation of $\mathrm{CO}_{2}$ at $157 \mathrm{~nm}$. Significant vibrational and rotational excitation of the daughter $\mathrm{CO}$ is observed. About $21 \%$ of CO is produced in the $v=1$ vibrational state and the rest in the $v=0$ state. The rotational population is observed to be distributed from $J=0$ to 42 for the $v=0$ state, from $J=0$ to 28 for the $v=1$ state. From these energy distributions and from the total excess energy for $\mathrm{CO}$ following $\mathrm{CO}_{2}$ photodissociation, we evaluate that the remaining energy, conveyed to the $\mathrm{CO}$ translational energy, corresponds to a $\mathrm{CO}$ ejection velocity of $0.8 \mathrm{~km} \mathrm{~s}^{-1}$. Indeed, Miller et al. observed such velocities from the Doppler profiles of the $\mathrm{CO}$ lines.

In cometary conditions, $\mathrm{CO}_{2}$ could dissociate from absorption at wavelengths still shorter than $157 \mathrm{~nm}$, giving more excess energy. Photodissociating $\mathrm{CO}_{2}$ could also be in a highly excited rotational state as a result of many IR pumping cycles throughout its long lifetime, increasing even more the available energy. So, the real CO ejection velocity could be even larger than $0.8 \mathrm{~km} \mathrm{~s}^{-1}$. Such a high velocity is definitely too large for accommodating our observed $\mathrm{CO}$ spectra, ruling out $\mathrm{CO}_{2}$ as a parent for $\mathrm{CO}$.

From the non-detection of any signal in the wings of the $\mathrm{CO}$ profile where $\mathrm{CO}$ produced by $\mathrm{CO}_{2}$ could show up, the coma model gives an estimate of $2 \times 10^{30} \mathrm{~mol} \mathrm{~s}^{-1}$ as a limit to the $\mathrm{CO}_{2}$ production, using the minimum ejection velocity of $0.8 \mathrm{~km} \mathrm{~s}^{-1}$. Given the possibility of even higher ejection

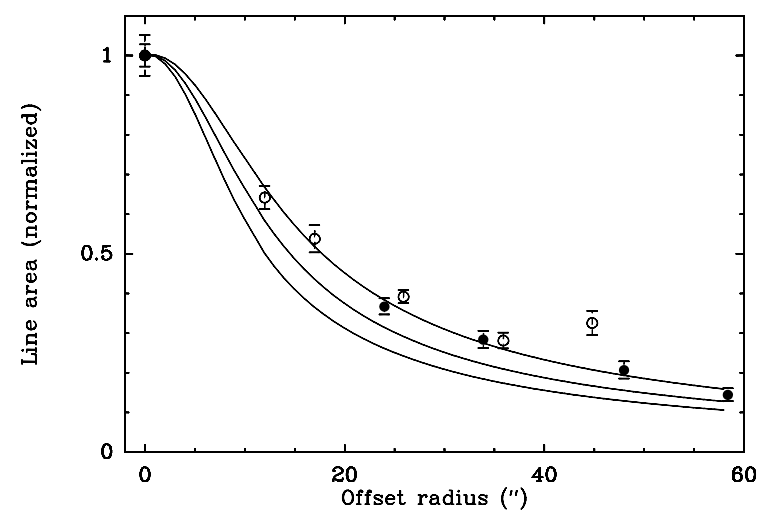

Fig. 7. Normalized line area of the radial average spectra shown (as in Fig. 5) for 2003 (open circles), and 2004 (filled circles) compared to that predicted by a model (solid line) assuming nuclear $\mathrm{CO}$ production and a cold inner coma. The temperature is (from bottom to top) 6, 5 , and $4 \mathrm{~K}$.

velocities, we infer that the $\mathrm{CO}_{2}$ production rate should not exceed a few $10^{30} \mathrm{~mol} \mathrm{~s}^{-1}$ in SW1.

We have considered mass loading of the gas flow by $\mathrm{CO}_{2}$ as an explanation of why the $\mathrm{CO}$ velocity is lower than that predicted by modelling (see Sect. 3.1). Such a process would require a minimum $\mathrm{CO}_{2}$ production rate of $\sim 2 \times 10^{29} \mathrm{~mol} \mathrm{~s}^{-1}$. This is well below the upper limit we find, and the idea of mass loading by $\mathrm{CO}_{2}$ can thus not be ruled out using the $\mathrm{CO}$ line shapes.

\subsubsection{Cold inner coma}

The observed radial evolution in line intensity appears to call for a zone in the innermost coma that is depleted in $\mathrm{CO}$ molecules compared to the outside region. But this does not necessarily mean that $\mathrm{CO}$ must be created by a coma source, as the observations include only those molecules that are "visible" to the telescope. For the present observations, the visible molecules are those that populate the $J=2$ rotational level. As the gas is released from the nucleus and expands into the coma, its temperature quickly drops through adiabatic cooling. If the kinetic temperature becomes low enough, most of the molecules will occupy the $J=0,1$ states, and be invisible to the telescope observing at $230 \mathrm{GHz}$. Also, at temperatures as low as a few $\mathrm{K}$, the effect of absorption of the $3 \mathrm{~K}$ background radiation becomes non-negligible, which reduces the intensity of the $230 \mathrm{GHz}$ line even more.

With an initial coma temperature of $8 \mathrm{~K}$, the fractional population of the $J=2$ level is 0.19 , and for $4 \mathrm{~K}$ it is only 0.04 . As the molecules travel outward, the population evolves toward fluorescence equilibrium, with a population of 0.27 in the $J=2$ level.

While we derived the value of $T_{\mathrm{k}}$ from the linewidth in Sect. 3.2.1, lower values for the temperature of the inner coma cannot be ruled out. The broadness of the blueshifted narrow peak only gives an upper limit to its value. The linewidth is also influenced by the outgassing pattern around the subsolar point and how it couples with the beam. It may accommodate lower temperatures, as shown in Sect. 3.4.

Figure 7 shows the radial behaviour of a coma with the same outgassing pattern as that shown in Fig. 5, but for inner coma temperatures of 6,5 and $4 \mathrm{~K}$. At the very low temperature of $4 \mathrm{~K}$, we see that an agreement with the observations, in terms of line area, can be reached. 
A
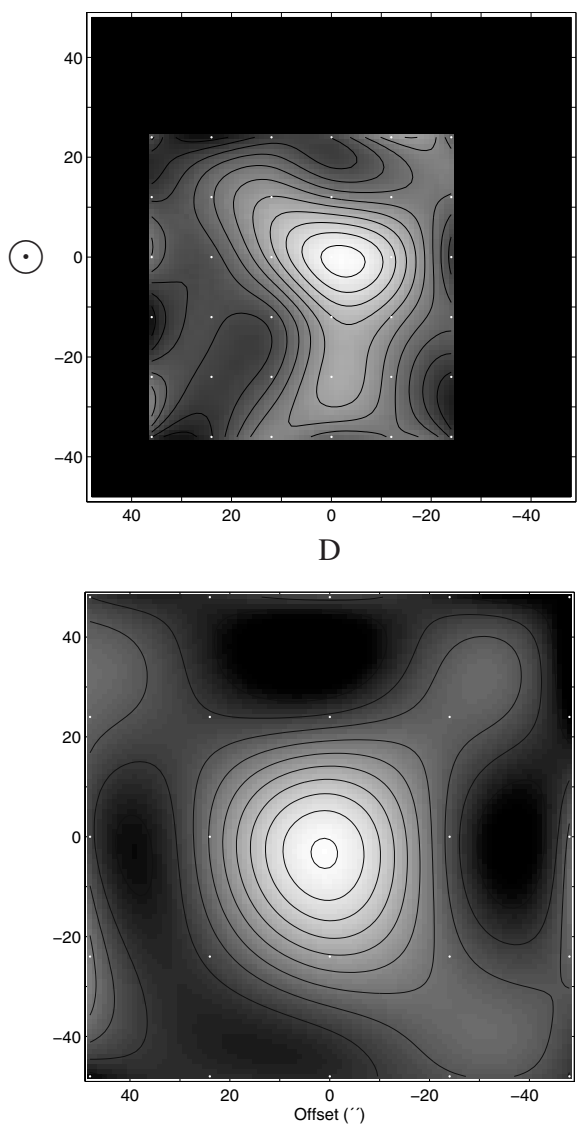

B
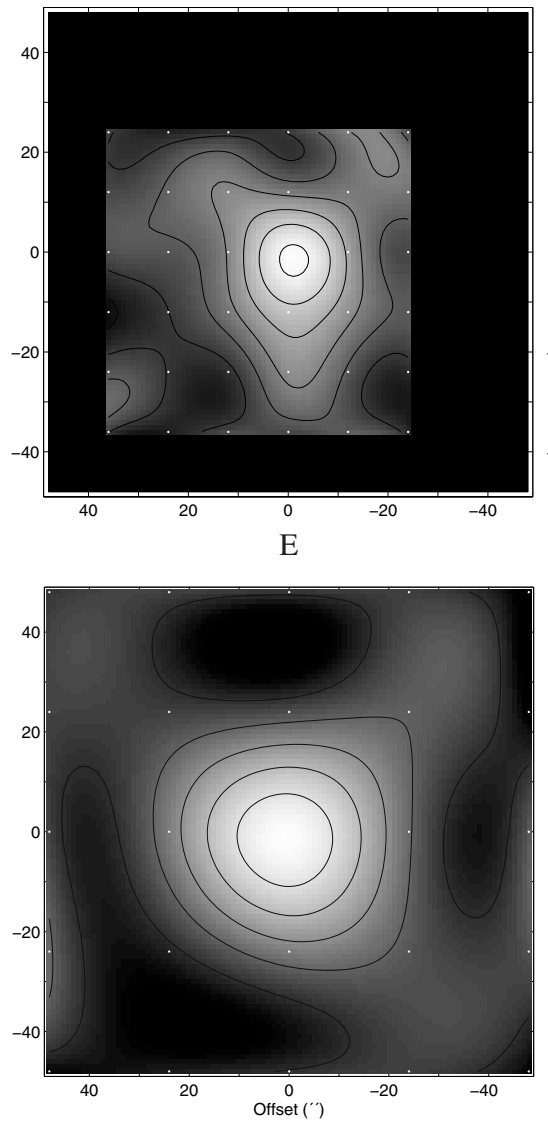

$\mathrm{C}$
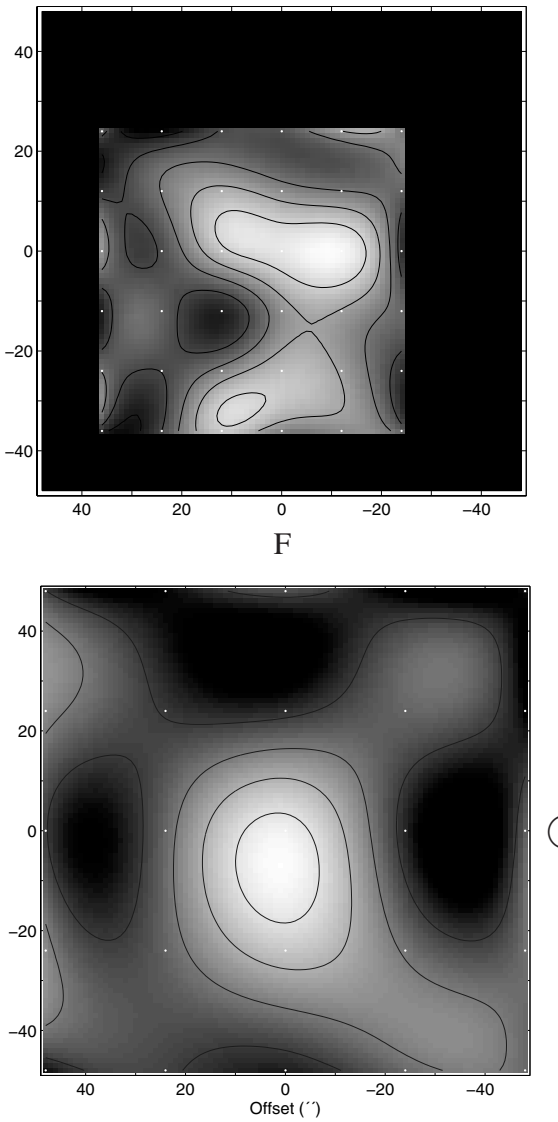

Fig. 8. The line area in the two datasets, for illustrative purposes displayed as re-sampled greyscale maps with contours. Each contour represents a $0.015 \mathrm{~K} \mathrm{~km} \mathrm{~s}^{-1}$ difference in line area. The orientation and layout of the maps correspond to Figs. 1 and 2 , with images A-C showing the June 2003 data and D-F showing the January 2004 data. Images A and D show the total line area, whereas B and E show only that of the narrow peak, and $\mathrm{C}$ and $\mathrm{F}$ show the area of the remainder of the line.

\subsection{Detailed character of the coma}

Having arrived at a general picture of how the radial intensity variation of the emission line is controlled by a cold inner coma, we proceed to look for asymmetries or anisotropies in the maps. In order to visualize the $2 \mathrm{D}$ intensity variation, Fig. 8 shows resampled greyscale maps, with contours. These images were produced through a 2D spline interpolation. Since they are based on a sparse grid of data points, they should be viewed with some caution, as small artificial features may appear. They are shown here for illustrative purposes, in order to give an impression of the most prominent coma features. The layout and scale of the greyscale maps is the same as for Figs. 1 and 2, and the mapped points are indicated by white dots. Maps A and D show the total line area for 2003 (A) and 2004 (D). From these two maps, the assumption of axial symmetry made when taking radial averages in the previous section appears valid for a global analysis.

Upon closer examination of maps A and D, we see that there exists an asymmetric feature common to the two. In a region about $30^{\prime \prime}$ south of the centre position in the 2003 map, the line intensity is stronger than in other positions at the same offset radius. The same feature is seen in the 2004 data, although only in the $24^{\prime \prime}$ south point. It is also possible to identify this region upon a careful viewing of the maps in Figs. 1 and 2.

While the main blueshifted peak of the spectra is well understood as outgassing from the nuclear dayside, the remainder of the line gave information about the distributed character of the CO production seen in the SEST spectra in 1998. In order to see if these two parts of the line have different characteristics when viewed in isolation we examine two additional maps, which are also shown in Fig. 8 for each dataset. Maps B and E show the line area of the main peak only, integrated between -0.7 and $-0.3 \mathrm{~km} \mathrm{~s}^{-1}$. In these two maps, we clearly see the concentration of emission at the central point, and axial symmetry prevails. Maps $C$ and $F$ then show the line area of the remainder of the line. In these, there is a marked asymmetry, and the region of excess emission to the south of the nucleus appears more clearly. Given the different values for $\Delta$ on the two observing runs, the conspicuous region is at the same absolute distance from the nucleus, i.e. $1.1 \times 10^{6} \mathrm{~km}$.

\subsection{Model fits and production rates}

\subsubsection{Model fit for the global case}

We remove the spectra in the region of "excessive" emission from the datasets (6 points for 2003 and 1 point for 2004), and re-calculate the radial average spectra for both data sets. With this adjustment, and with the derived value of $4 \mathrm{~K}$ for the inner coma temperature, we carefully adjust the outgassing pattern in order to perform fits of the model line profile to the centremost spectra for the two datasets. The coma model then produces simulated line profiles that agree with the observed line shapes and intensities at all offset radii. The case for 2004 is shown in Fig. 9.

In the framework of this coma model, described in detail in Gunnarsson et al. (2002), the outgassing pattern is determined 

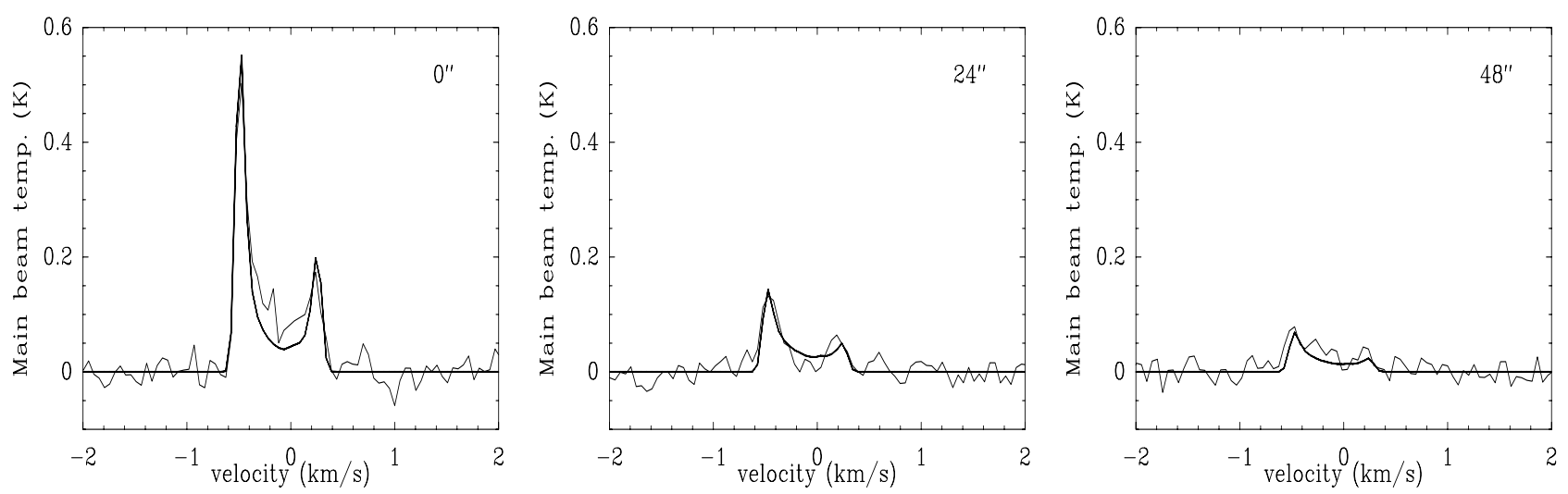

Fig. 9. The radial average CO line (thin line) at $0^{\prime \prime}, 24^{\prime \prime}$ and $48^{\prime \prime}$ from the 2004 dataset (excluding the point $24^{\prime \prime}$ south), compared to the model line (thick line) assuming an inner coma temperature of $4 \mathrm{~K}$.

Table 3. Outgassing patterns found for the best model fits to the two datasets, with an inner coma temperature of $4 \mathrm{~K}$.

\begin{tabular}{lll}
\hline \hline & 2003 & 2004 \\
\hline Subsolar gas velocity $\left(\mathrm{m} \mathrm{s}^{-1}\right)$ & 505 & 510 \\
Nightside gas velocity $\left(\mathrm{m} \mathrm{s}^{-1}\right)$ & 280 & 280 \\
CO production night/day contrast & 0.22 & 0.23 \\
\hline
\end{tabular}

by three free model parameters $a, b$ and $c$, and $\mu$, defined in Sect. 3.2.1. The outgassing velocity field is defined as $v=a \mu+b$, with $b$ being the nightside velocity and $a+b$ the subsolar velocity. The local production rate is proportional to $\mu^{2}+c$. The contrast in night/day production given here is the ratio of the total rates for the nightside and dayside hemispheres. The outgassing patterns derived from the best model fits are almost identical, and their features are listed in Table 3. The night/day production ratio is similar to that observed at SEST in 1996, when it was 0.25 (Festou et al. 2000).

The total $\mathrm{CO}$ production rate is derived as a direct result from the model fitting procedure, as this is also a free model parameter. As the coma model now explains both the shape and the area of all the observed emission lines in the global study, we are able to present nuclear production rates of $\mathrm{CO}$ that are based on an improved insight into the nature of SW1. For June 2003, $Q_{\mathrm{CO}}$ was $(3.9 \pm 0.2) \times 10^{28} \mathrm{~mol} \mathrm{~s}^{-1}$, and for January 2004 it was $(3.7 \pm 0.2) \times 10^{28} \mathrm{~mol} \mathrm{~s}^{-1}$. As a large fraction of the molecules are not in the $J=2$ state in the inner coma, the derived values of $Q_{\mathrm{CO}}$ are about a factor $\sim 1.5$ greater than what would result from a calculation based on a model that uses the nucleuscentred spectra only.

\subsubsection{The "excessive" region}

Comparing then the spectra from the removed region only, to the line predicted by the above model fit for this position (Fig. 10), we see that the excess emission has an identical character on the two occasions. The difference in beam temperature is roughly constant inside the edges of the line. As described in Sect. 3.2.2, such a symmetric addition is what is expected for an extended source consisting of icy grains, such as that suggested in Gunnarsson et al. (2002). The average velocity shift of the spectra in the removed region is $-0.18 \pm 0.02 \mathrm{~km} \mathrm{~s}^{-1}$ (2003) and $-0.17 \pm 0.03 \mathrm{~km} \mathrm{~s}^{-1}(2004)$, i.e. a smaller shift than the average of Fig. 6.
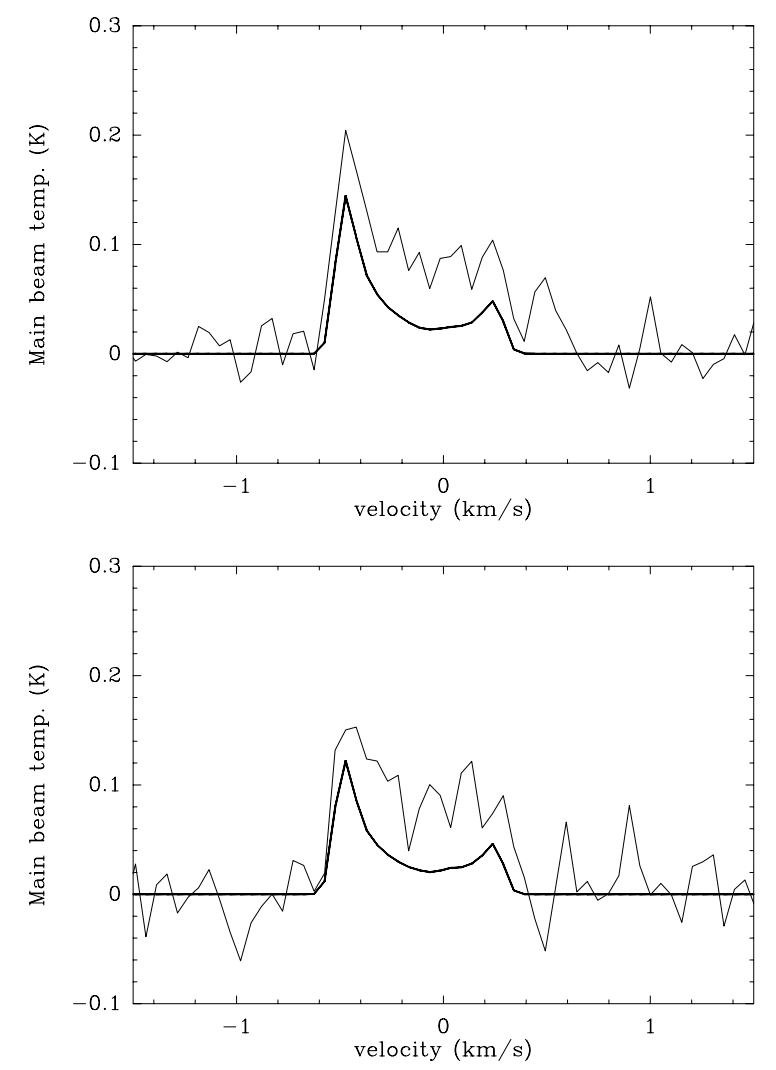

Fig. 10. The emission line in a region around $30^{\prime \prime}\left(=1.1 \times 10^{6} \mathrm{~km}\right)$ south of the centre position for 2003 (above) and 2004 (below), at the same distance, compared to the model prediction using a cold inner coma. An excess is seen in the observed lines, with a similar appearance in both occasions.

Although the effect seen in Fig. 10 is not presented here as absolute proof of the presence of such a source in the new data, it shows that the striking extended feature that completely covered the 1998 map observed at SEST is not entirely absent in the new data.

An enhancement in dust density in this direction has been seen in the infrared using the Spitzer telescope (Stansberry et al. 2004) in observations between the two HERA observations. This may hint at a connection between the dust coma and the possible $\mathrm{CO}$ source, but the confinement of such a source in a limited region is not easily explained. 


\section{Conclusion}

In visual observations, it is the strongly variable brightness of comet SW1 that has made it famous. We know from earlier observations (Biver 1997) that the CO production rate varies from time to time by up to a factor 3 , and also that the velocity of the blueshifted peak can vary between -0.45 and $-0.50 \mathrm{~km} \mathrm{~s}^{-1}$. In the present work, however, we took two "snapshots" of the activity of the comet separated by half a year, during which almost no change in any aspect of the $\mathrm{CO}$ production can be seen.

The maximum gas velocity of $0.50 \mathrm{~km} \mathrm{~s}^{-1}$ is lower than that expected from theoretical models (Sect. 3.1). The reason for this lower velocity could be mass loading by $\mathrm{CO}_{2}$, a process that cannot be ruled out using the upper limit to $\mathrm{CO}_{2}$ production that we find from the $\mathrm{CO}$ line shapes.

The large extended CO source, which was suggested by the SEST data from 1998 (Gunnarsson et al. 2002) to dominate the $\mathrm{CO}$ production, and which was to be the focus in the present project, is not seen in 2003-2004. A constant line area up to $22^{\prime \prime}$ offset, as observed in 1998, would not be explained by excitation effects, nor by a parent molecule, as these scenarios always give a maximum at the centre of the map. While the 1998 observations indicate the presence of a large extended source, we now see that it is not a permanent feature of the comet, but rather may have represented extraordinary conditions. Still, as indicated in Figs. 8 and 10 the signature of the extraordinary observations from 1998 may not be completely absent in the new data.

The very low temperature of $4 \mathrm{~K}$, which is needed to explain the radial profile in the new data, is extreme, but not unrealistic. Hydrodynamical modelling work (Crifo et al. 1999), done for the specific case of SW1, points to gas temperatures close to this value. This work is valid up to a distance where translational relaxation occurs, but for anisotropic outgassing, the kinetic temperature is already below $6 \mathrm{~K}$ at this distance. Using a Monte Carlo model, the gas in SW1 has been simulated outside the point where translational relaxation begins, and very low rotational temperatures $(2-3 \mathrm{~K})$ were predicted (V. Zakharov, pers. communication).

If a simultaneous observation of the $J=2-1$ and $J=1-0$ emission lines of $\mathrm{CO}$ is done with a $\mathrm{S} / \mathrm{N}$ ratio high enough, the temperature of the inner coma may be determined well enough to further support or disprove the low temperature prediction. The $(2-1) /(1-0)$ line area ratio resulting from the best fit using our model is 3.17 , which is well within the limits of the value $3.26 \pm 0.43$, calculated from a long series of accumulated observations by Biver (1997).

As the low temperature we find makes much of the $\mathrm{CO}$ gas in the innermost coma "invisible" at $230 \mathrm{GHz}$, a larger nuclear production rate is required to explain the observed spectrum. Naturally, this applies also to the values previously published. The production rate is thus about 1.5 times higher than the value derived from the shape and intensity of a nucleus-centred emission line only.

The present case shows that comets indeed provide a laboratory for the study of gases at extremely low densities and temperatures - and comet SW1, with its near-circular orbit, is always available for us to investigate.

Acknowledgements. This paper is dedicated to our colleague and team member Michel Festou (1945-2005), and we wish to acknowledge his many years of expertise and dedicated research, and his important role in the study of comet SW1 in particular. The work done by Gunnarsson was supported through a fellowship from Université Pierre et Marie Curie, Paris VI.

\section{References}

Biver, N. 1997, Ph.D. Thesis, Université Paris VII

Biver, N., Bockelée-Morvan, D., Crovisier, J., et al. 2007, Planet. Space Sci., 55, 1058

Bockelée-Morvan, D., Crovisier, J., \& Gerard, E. 1990, A\&A, 238, 382

Cabot, H., Enzian, A., Klinger, J., \& Majolet, S. 1996, Planet. Space Sci., 44, 1015

Crifo, J. F., \& Rodionov, A. V. 1997, Icarus, 127, 319

Crifo, J. F., Rodionov, A. V., \& Bockelée-Morvan, D. 1999, Icarus, 138, 85

Crovisier, J., Biver, N., Bockelée-Morvan, D., et al. 1995, Icarus, 115, 213

Crovisier, J., Leech, K., Bockelée-Morvan, D., et al. 1997, Science, 275, 1904

Enzian, A., Cabot, H., \& Klinger, J. 1997, A\&A, 319, 995

Festou, M. C., Gunnarsson, M., Winnberg, A., Rickman, H., \& Tancredi, G. 2000, Icarus, 150, 140

Gunnarsson, M., Rickman, H., Festou, M. C., Winnberg, A., \& Tancredi, G. 2002, Icarus, 157, 309

Huebner, W. F., Keady, J. J., \& Lyon, S. P. 1992, Ap\&SS, 195, 1

Miller, R. L., Kable, S. H., Houston, P. L., \& Burak, I. 1992, J. Chem. Phys., 96, 332

Schuster, K.-F., Boucher, C., Brunswig, W., et al. 2004, A\&A, 423, 1171

Senay, M. C., \& Jewitt, D. 1994, Nature, 371, 229

Stansberry, J. A., Van Cleve, J., Reach, W. T., et al. 2004, ApJS, 154, 463

Tancredi, G., Rickman, H., \& Greenberg, J. M. 1994, A\&A, 286, 659 